\title{
CLASSIFICATION OF MIXED ODORS USING A LAYERED NEURAL NETWORK
}

\author{
Sigeru Omatu \\ Osaka Institute of Technology, \\ 1-45 Chayamachi Kita-ku, Osaka, 530-8568, Japan \\ omatu@rsh.oit.ac.jp, https://www.oit./rd/
}

\begin{abstract}
Odor classification has been forcused due to one of five senses of human being. If we could establish the odor classification technology, we would expect various new technology since human being requires five sences to acheive higher quality information processing and sophistcated decision making. For example, we could expect the odor classification and odor synthesis, which enable us to achieve odor communication technology. Furthermore, the odor classification would be applicable to keep the society safe by detecting the dangerous odors and to make our life more satisfactory by using additional odor information. In this paper we develop an electronic nose using a neural network. The neural network is a multi-layered neural network based on the gradient method. After classifying the various odors, we consider the classification in case that mixed odors are measured. To improve the classification accuracy we adopt a genetic algorithm to find a reduction factor to separate two mixed odors. Copyright $\mathbb{C}$ Research Institute for Intelligent Computer Systems, 2017. All rights reserved.
\end{abstract}

Keywords: odor feature vector: layered neural networks, separation of mixed gasses: odor classification.

\section{INTRODUCTION}

The 2004 Nobel Prize in Medicine and Physiology was awarded to Richard Axel and Linda B. Buck who clarified the mechanism of odor receptors for the paper of [1]. They clarified the actual state of the protein and pursued how this protein sends information in the brain [2]. For human beings it is thought that there is the ability to split 10,000 kinds of things into you, from the meat of the lover to the perfume of the lover.

Odor is one of five senses for human and it makes our life pleasant or unpleasant based on odor environment. Odor study has been developed from chemical sensing viewpoint [3]-[10]. Not only chemical sensors but also semiconductor sensors have been developed [11]-[14].

Odor study becomes very important to make the human life comfortable. Based on the progress of medical research on nose system, artificial electronic nose (E-nose) systems have been paid much attention. The many E-nose systems from commercial and technical viewpoints are quality control of food and public safety [14]-[19]. We will discuss a new E-nose system to separate two kinds of odors from mixed odors by using a layered neural network. J. A. Milke [20] is the first person who used two kinds of metal-oxide semiconductor gas sensor (MOGS) to classify several sources for fire. His results were only $85 \%$ of correct classification based on a conventional statistical pattern recognition.

By using neural networks we have developed for odor classification of various sources for fire such as household burning materials in [21]. In this paper we focuses on mixed odors classification. Various odors have been mixed naturally in our living environment. If a poisonous odor exists in our living environment, it must be recognized immediately by humans. Therefore, it is necessary to classify mixed odors into each component of mixed odors. First, we have measured mixed odors as well as each odor before mixing. After that we train the neural network to classify each component precisely. Finally, we use the trained neural network to classify the mixed odors into original odor using the method of [22].

\section{OLFACTORY MECHANISM}

A human olfactory system has not been fully understood. But by physicians, the some knowledge about the anatomy of human olfactory system has been made clear. Some of them are the olfactory epithelium, the olfactory bulb, the olfactory cortex, and the higher brain or cerebral cortex. Odor classification process of human is as follows: 
Step 1. Sniffing odorous molecules to a nose.

Step 2. They are dissolved and detected by olfactory receptors

Step 3. Signals from olfactory receptors are transported olfactory bulbs.

Step 4. Olfactory bulb transforms the information of olfactory bulb to olfactory cortices.

Step 5. Information of olfactory cortices is distributed to other parts of brain and human can identify the odor.

Step 6. Cleaning the nose by breathing fresh air.

Olfactory mechanism is shown in Fig. 1. Organisms have olfactory receptor cells which gather in the myriad. Holes which respond to a particular molecule have been opened to each olfactory receptor. If the odor substances that correspond the keyhole came, the odor substances are made to react nerve. The olfactory cells are converted into an electrical signal. Then an electrical signal is send to the brain through the olfactory bulbs. In this way, living organisms sniff out odors. Humans have about 350 kinds of olfactory receptor.

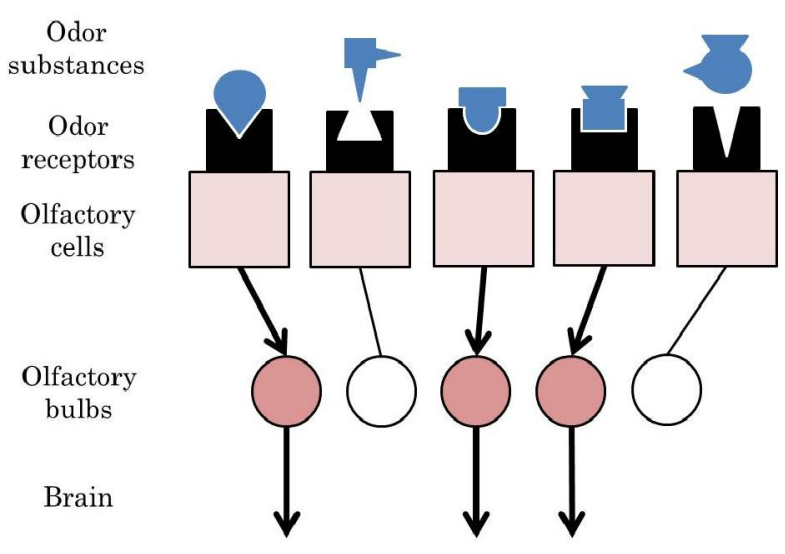

Fig. 1 - Working olfactory cells

\section{ODOR SENSORS AND SENSING SYSTEM}

Gas sensors using a tin oxide was produced in 1968. There are tin, iron oxide, and tungsten oxide as typical gas sensing substances which are used in metal oxide semi-conductor gas sensors (MOG sensor).

Provide sufficient detail to allow the work to be reproduced. We explain about mechanism of the gas sensor to detect odor substances.

First step is to heat a metal oxide semiconductor gas sensor by about 300 degrees Celsius, which means an oxidation reaction such that

$$
\frac{1}{2} \mathrm{O}_{2}+\left(\mathrm{SnO}_{2-\mathrm{x}}\right)^{*} \Rightarrow \mathrm{O}^{-} \mathrm{ad}\left(\mathrm{SnO}_{2-\mathrm{x}}\right)
$$

where ad( ) shows adsorption of ( ) and an electrical resistance of the tin becomes high.

On the other hand, when a gas, for example $\mathrm{CO}$ gas, will come, the following reduction occurs

$$
\mathrm{CO}+\mathrm{O}^{-} \mathrm{ad}\left(\mathrm{SnO}_{2-\mathrm{x}}\right) \Rightarrow \mathrm{CO}_{2}+\left(\mathrm{SnO}_{2-\mathrm{x}}\right)^{*} .
$$

In this case, an electrical resistance of the tin becomes low. Roughly speaking, oxygen and reduction occur and the metal-oxide gas sensor becomes variable resistance.

Using the above oxidation and reduction process, we could measure whether a gas appears or not as shown in the Fig. 2. MOG sensors need to be operated at high temperature. Thus, they consume a little higher power supply than the other kinds of sensors. The reliability and the sensitivity of MOG sensors are proved to be good to detect volatile organic compounds (VOCs), combustible gas, and so on.

However, the choices of MOG sensors are still not cover all odorous compounds and it is difficult to create an MOG sensor that responds to one odor precisely. Generally, most commercial MOG sensors respond to various odors in different ways. Therefore, we can expect if we use many MOG sensors to measure odor, the vector data reflect the specific properties for the odor[21].

Generally, it is designed to detect some specific odor in electrical appliances such as an air purifier, a breath alcohol checker, and so on. Each type of MOG sensors has its own characteristics in the response to different gases. When combining many MOG sensors together, the ability to detect odor is increased. The main part of the MOG sensor is the metal oxide element on the surface of the sensor. When this element is heated at certain high temperature, the oxygen is adsorbed on the crystal surface with the negative charges.

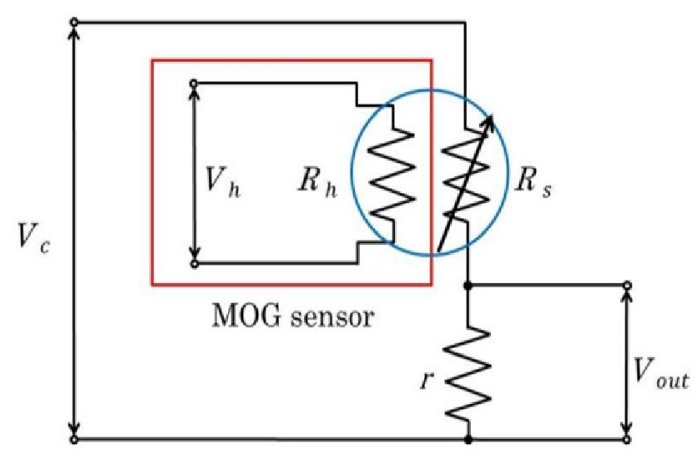

Fig. 2 - Odor sensing circuit

The reaction between the negative charge of the metal oxide surface and deoxidizing gas makes the 
resistance of the sensor vary as the partial pressure of oxygen changes. Based on these characteristics, we can measure the net voltage changes while the sensors adsorb the tested odor. In this paper, MOG sensors are used to measure the single odor and mixed odor. Table 1 shows the five sensors in the experiment.

Table 1. Gas sensors used in the experiment used in the experiment.

\begin{tabular}{|l|l|l|}
\hline Sensor & Gas detection & Applications \\
\hline 1.SB-AQ8 & volatile & air quality \\
\hline 2. SB-15 & propane & flammable gas \\
\hline 3. SB-42A & freon & refrigerant gas \\
\hline 4. SB-31 & organic solvent & solvent \\
\hline 5. SB-30 & alcohol & alcohol \\
\hline
\end{tabular}

\section{PATTERN RECOGNITION PROCESS}

The pattern recognition is corresponded the observed pattern. This concept is called a class or category. For example, in the case of alphabet recognition, the classification processing corresponds to one of the 26 classes of input patterns. When we constitute a pattern recognition system, it is generally uses the form of Fig. 3 as typical pattern recognition processing procedure. The characteristics extraction unit extracts characteristics from the input original signal. The classification is performed by using those characteristics. It is necessary to learn those characteristics. Then the classification is performed by matching with the training data.

\section{NEURON MODEL}

We show the neural network fundamental structure in Fig. 4. The left hand side denotes input $\left(x_{1}, x_{2}, \ldots, x_{n}\right)$, the right hand side $y_{j}$ is the output, $w_{j i}(i=0,1, \ldots, n)$ shows weighting coefficient, and $f(\cdot)$ is an output function of the neuron, Note that $x_{0}=-1$ and $w_{j 0}=\theta_{j}$ where $\theta_{j}$ shows a threshold of the neuron $j$. There are several types of the output function $f(\cdot)$. We adopt the sigmoid function given by Fig. 5 .

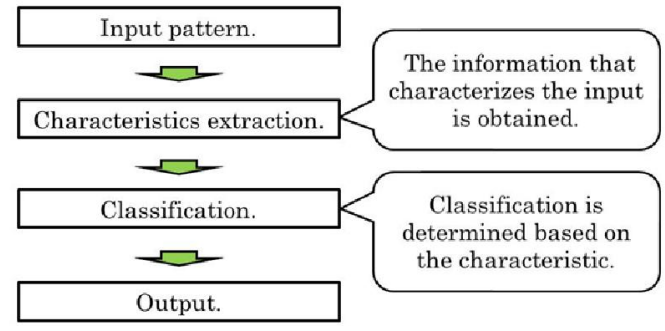

Fig. 3 - Typical pattern classification process

\section{ERROR BACK-PROPAGATION METHOD}

We use the layered neural network. Neural networks are mainly used in pattern recognition, associative memory such as text and voice. Neural network is classification as supervised learning and unsupervised learning. When definite answer need, we use the supervised learning. In addition, when disparate data divide into several groups, we use unsupervised learning. The characteristics of the layered neural network are shown below. The layered neural network has input layer, hidden layer, and output layer as shown in Fig. 6.

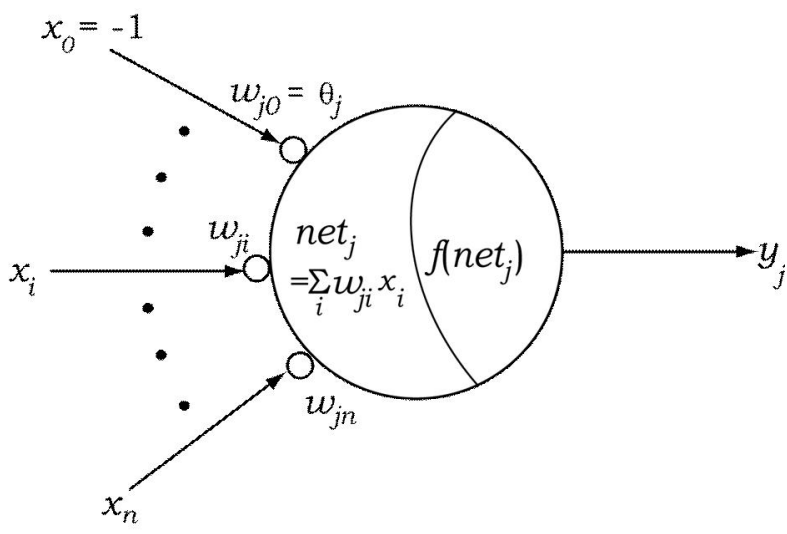

Fig. 4 - Neuron model

There is an error back-propagation method which is one of the learning methods of the layered neural network. After forward calculations, we calculate mean squared error of each output value and desired values. Neural network modifies weighting coefficients of each neuron by using the error. The mean squared error would be minimized until it becomes minimum based on gradient method. Error back-propagation is the most widely used of neural network paradigms and has been applied successfully in applications studies in a broad range of areas. It is one of the easiest networks to understand. If desired output $d_{k},(k=1,2, \ldots, K)$ are assigned in Fig. 6 , the error $e_{k},(k=1,2, \ldots, K)$ occurs. Then the weighting coefficients, $w_{j i} \quad(i=0,1, \ldots, I, j=1,2, \ldots, J) \quad$ and $\quad w_{k j}$, $(j=0,1, \ldots, I, k=1,2, \ldots, K)$ are corrected such that the error becomes smaller based on the gradient method.

If the number of hidden layer is increased, pattern recognition ability improves. However, advantage of high speed calculation lost. The way of calculation is shown below. Note that the number of neurons at input layer is $\mathrm{I}$, the number of neurons at hidden layer is $\mathrm{J}$, and the number of output layer is $\mathrm{K}$. 


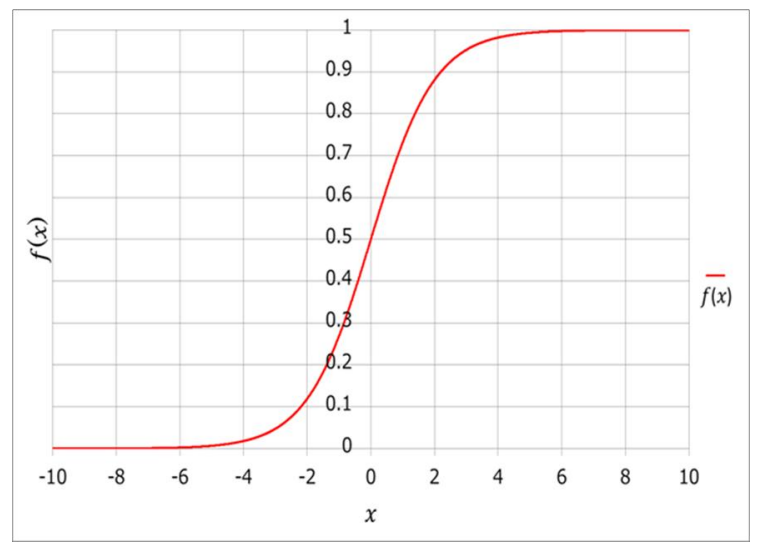

Fig. 5 - Sigmoid function

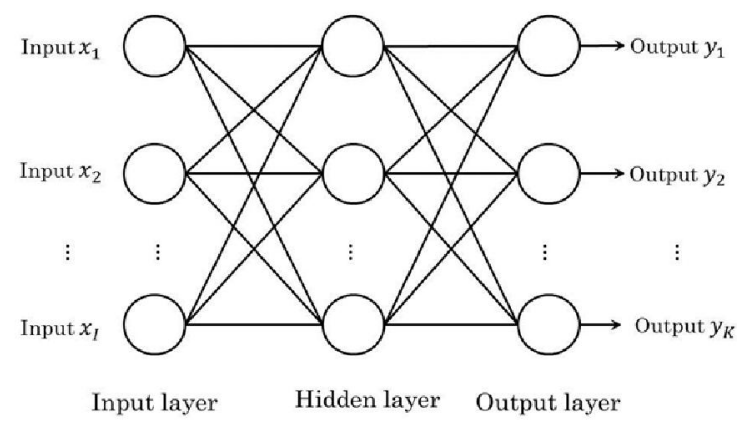

Fig. 6 - Layered neural network

In order to classify the odors we adopt a threelayered neural network based on the error backpropagation method. The error backpropagation algorithm is given by the following steps.

Step 1. Assign the initial values of $w_{j i}, w_{k j}$, and $\eta>0$.

Step 2. Assign $d_{k},(k=1,2, \ldots, K)$ the reference values of output $y_{k},(k=1,2, \ldots, K)$, corresponding to the input data $x_{i},(i=1,2, \ldots I)$.

Step 3. Calculate

$$
\begin{aligned}
& \text { net }_{j}=\sum_{i=0}^{I} w_{j i} x_{i}, O_{j}=f\left(\text { net }_{j}\right), \\
& \text { net }_{k}=\sum_{j=0}^{J} w_{k j} O_{j}, O_{k}=f\left(\text { net }_{k}\right) .
\end{aligned}
$$

Step 4. Calculate the error $e_{k}$ and generalized error

$$
\begin{aligned}
& \delta_{k}, e_{k}=d_{k}-y_{k}, \\
& \delta_{k}=e_{k} O_{k}\left(1-O_{k}\right), \\
& \delta_{j}=\sum_{k=1}^{K} w_{k j} \delta_{k} O_{j}\left(1-O_{j}\right) .
\end{aligned}
$$

Step 5. If $e_{k}$ is sufficiently small, END. Otherwise,

$$
\begin{aligned}
\Delta w_{k j} & =\eta \delta_{k} O_{j} \\
\Delta w_{j i} & =\eta \delta_{j .} . O_{i} .
\end{aligned}
$$

Step 6.

$$
\begin{aligned}
& w_{k j}=w_{k j}+\Delta w_{k j} \\
& w_{j i}=w_{j i}+\Delta w_{j i} .
\end{aligned}
$$

Step 7.

Go to Step 3.

Following the above recursive calculation, we can train the neural network. The measurement data are five dimensional vectors which are obtained with five sensors stated in Table 1.

\section{MEASUREMENT OF ODOR DATA}

We have measured four types of odors as shown in Table 2. Note that those factors in Table 2 are main odors included in our breath. The sampling interval is $200[\mathrm{~ms}]$, that is, the sampling frequency is $5[\mathrm{~Hz}]$. The temperatures of odor gases were 18 $24\left[{ }^{\circ} \mathrm{C}\right]$, and the humidity of gases is $20-30$ [\%]. We show a measurement cycle in Fig. 7 and the sample paths in Figs. 8-11. Bad odor of our breath will make the neighboring persons unpleasant although the person himself might not be aware of his smell.

Furthermore, the bad odor of persons may reflect a kind of diseases and the measurement of human breath and odor classification will be profitable to predict a disease of persons in advance.

Table 2. Substance of training data.

\begin{tabular}{|l|l|c|}
\hline Label & substance & no. of samples \\
\hline A & acetaldehyde & 3 \\
\hline B & ethylene & 3 \\
\hline C & methyl-mercaptan & 3 \\
\hline D & hydrogen sulfide & 3 \\
\hline
\end{tabular}

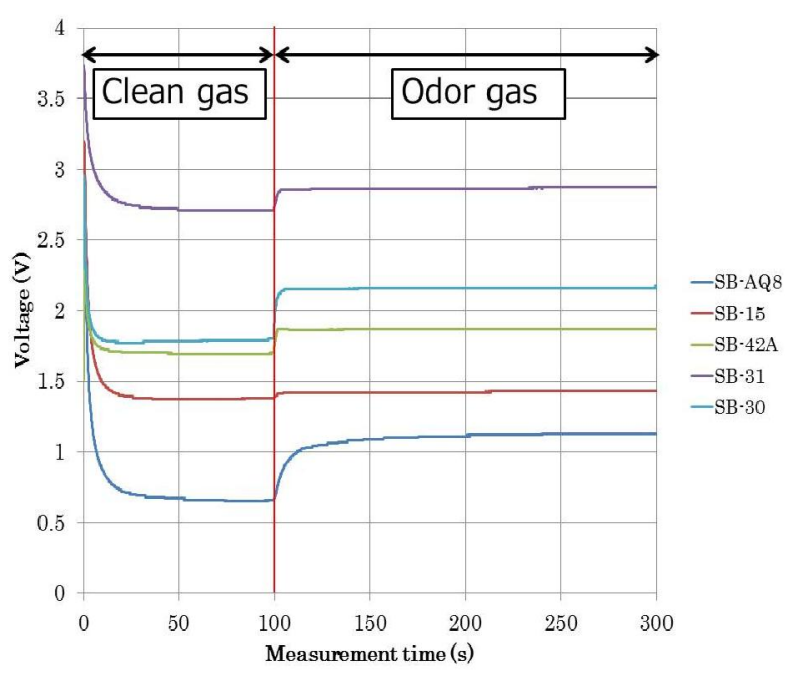

Fig. 7 - Clean gas and odor gas timing

The breath includes many gasses although the levels are very thin. To make the similar situation we mixed main gasses shown in Figs. 8-11 and regarded the mixed gasses as our breath. The density 
of these gasses is very thin and we made mixed gasses of $1 \mathrm{ppm}-5 \mathrm{ppm}$ order. The mixing gasses are $1: 1,1: 5$, and $5: 1$ mixing rates where $a: b$ shows that a ppm and $b$ ppm gasses are mixed. In case of four sources, there are six combination cases to mix two gasses.

The breath includes many gasses although the level are very thin. To make the similar situation we

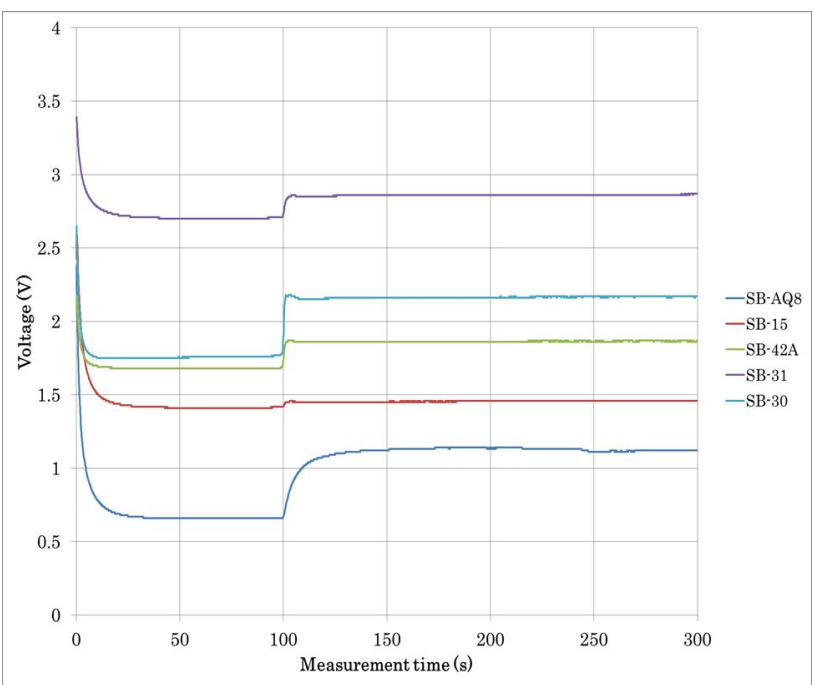

Fig. 8 - Sample path of acetaldehyde

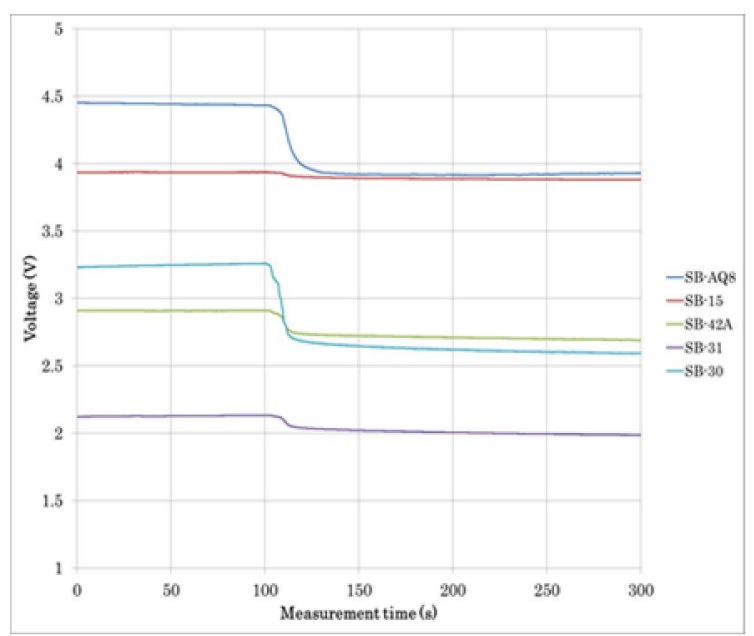

Fig. 10 - Sample path of methyl-mercaptan

\section{TRAINING FOR CLASSIFICATION}

In this paper, we have mixed only two gasses of methyl-mercaptan (C) and hydrogen sulfide (D) since they are main components in our breath. Those sample paths are shown in Figs. 12-14 for different mixing rates of methyl-mercaptan (C): hydrogen sulfide (D) such as $1: 1,1: 5$, and 5:1, respectively. Note that the voltages shown in Fig. 12-14 are upside down since we have measured the voltages between sensor teminals instead of measurering $V_{\text {out }}$ in Fig. 2, that is, we have measured $V_{c}-V_{\text {out }}$. mixed main gasses shown in Figs. 8-11 and regarded the mixed gasses as our breath. The density of these gasses is very thin and we made mixed gasses of $1 \mathrm{ppm}-5 \mathrm{ppm}$ order. The mixing gasses are 1:1, 1:5, and 5:1 mixing rates where $a: b$ shows that a ppm and $b$ ppm gasses are mixed. In case of four sources, there are six combination cases to mix two gasses.

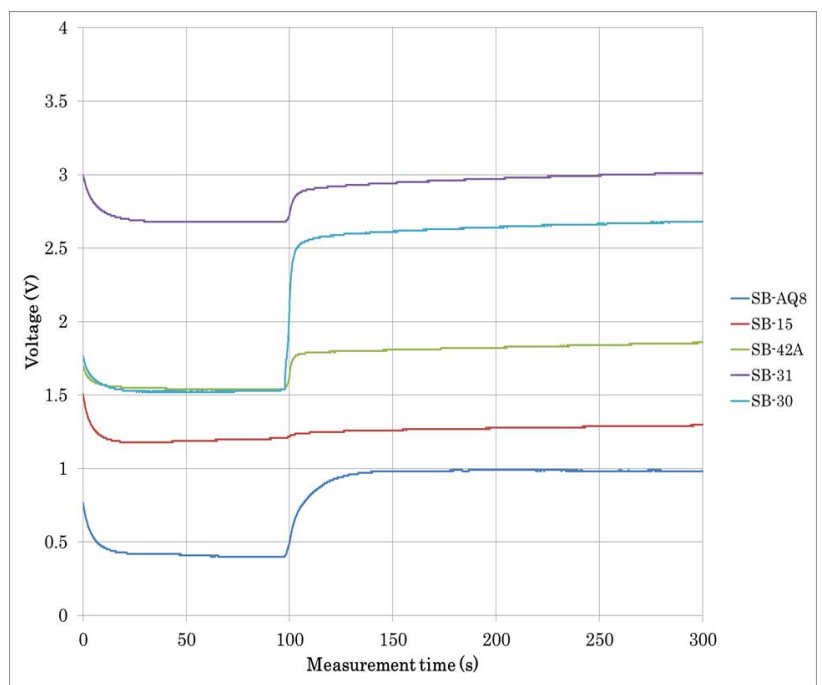

Fig. 9 - Sample path of ethylene

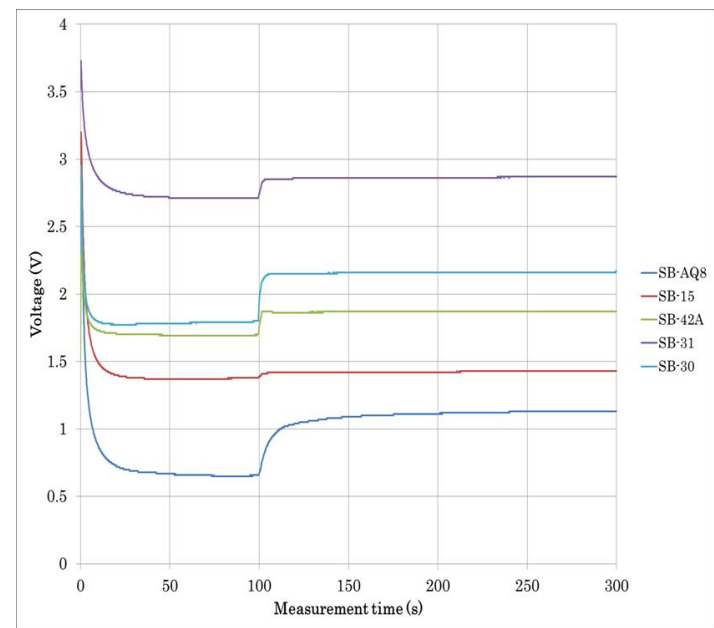

Fig. 11 - Sample path of hydrogen sulfide

In order to classify the feature vector, we allocate the desired output for the input feature vector where it is five-dimensional vector as shown in Table III since we have added the coefficient of variation to the usual feature vector to reduce the variations for odors. The training has been performed until the total error becomes less than or equal to $5 \times 10^{-3}$ where $\eta=.2$. Note that the training data set is the first sample data among three repeated data of A, B, $\mathrm{C}$, and D. After that, the second sample data are selected as the training data set and the third samples data set. 


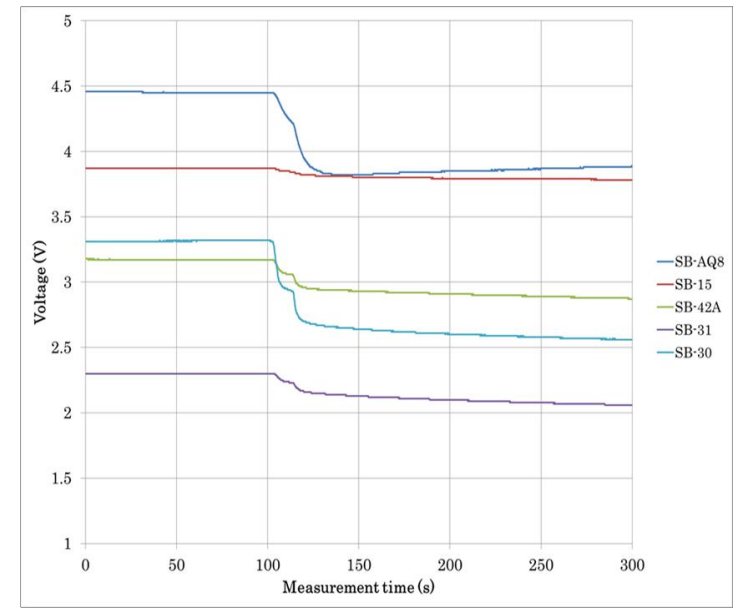

Fig. 12 - Sample path of mixing rate 1:1

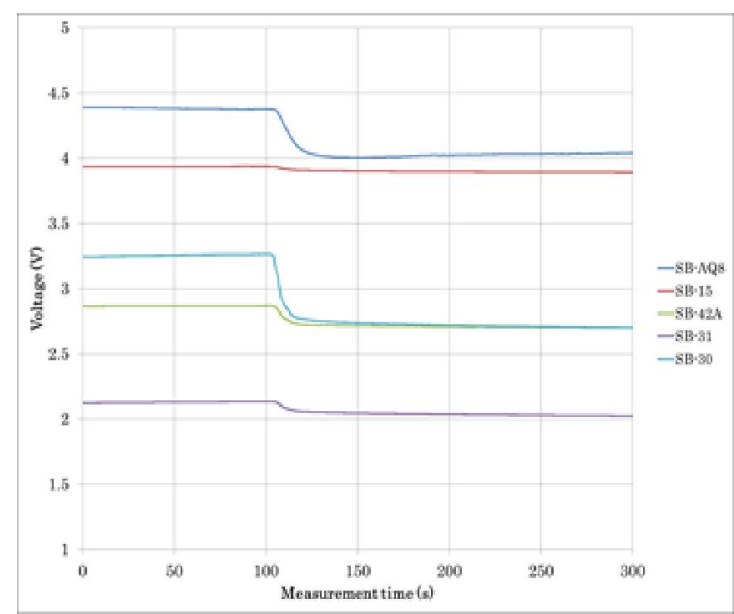

Fig. 13 - Sample path of mixing rate 1:5

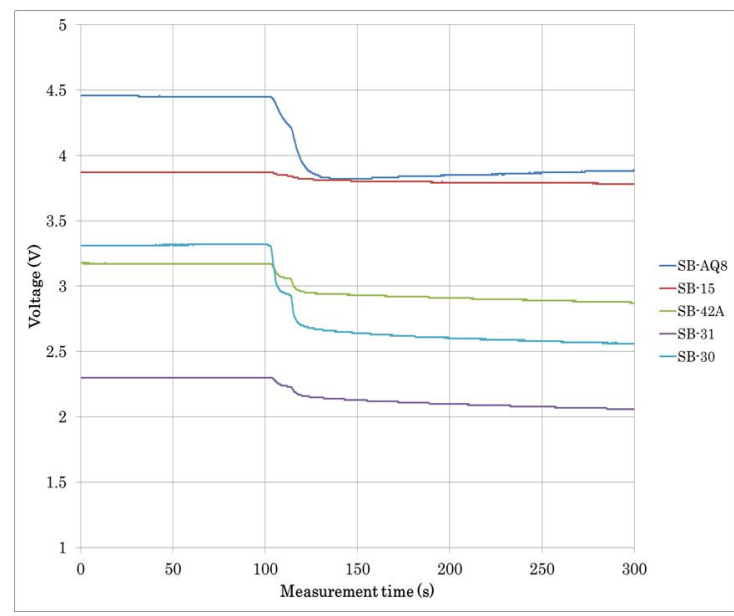

Fig. 14 - Sample path of mixing rate 5:1.

\section{CLASSIFICATION RESULTS FOR TEST DATA}

\subsection{SINGLE ODOR CLASSIFICATION RESULTS}

Using a layered neural network with five inputs and four output, we have trained the neural network for one training data until the total error becomes 0.001. After training, we checked the remaining two data set. For two test data, correct classification rates became $100 \%$ and using the leave-one-out cross validation check, correct classification rates were $100 \%$.

\subsection{MIXED ODOR CLASSIFICATION RESULTS}

Using the trained neural network, we tested the classification results whether the original two odors could be classified or not. The classification results are shown in Table IV. From this results, the classification results are not so good since there are many misclassification results came out. Especially, C\&D has been classified acetaldehyde, (A) because hydrogen sulfide (D) is too strong chemical reaction even if the very thin density levels as well as acetaldehyde (A). Thus, we must reconsider our method to classify the mixed odors classification. One of the approaches is to reduce the maximum values of odor data in order to prevent a sole winner case. Therefore, we use the following formula to reduce the effect of a sole winner.

Table 3. Training data set for acetaldehyde (A), ethylene (B), methyl-mercaptan (C), and hydrogen sulfide (D).

\begin{tabular}{|c|c|c|c|c|}
\hline labels & A & B & C & D \\
\hline A & 1 & 0 & 0 & 0 \\
\hline B & 0 & 1 & 0 & 0 \\
\hline C & 0 & 0 & 1 & 0 \\
\hline D & 0 & 0 & 0 & 1 \\
\hline
\end{tabular}

Table 4. Classification results for mixed odors of methyl-mercaptan $(C)$ and hydrogen sulfide(D).

\begin{tabular}{|c|c|c|c|c|}
\hline mixing rates & A & B & C & D \\
\hline $1: 1($ C\&D $)$ & 4 & 2 & 6 & 6 \\
\hline $1: 5(\mathrm{C} \& D)$ & 9 & 0 & 0 & 9 \\
\hline $5: 1(\mathrm{C} \& D$ & 9 & 0 & 0 & 9 \\
\hline
\end{tabular}

$$
z=x-\alpha y
$$

where $x$ is a feature, $\mathrm{y}$ is the top value of each row Table IV and a is a constant. If we set $\alpha=0.3$, we have the results of Table V.

Table 5. Classification results for mixed odors of methyl-mercaptan (C) and hydrogen sulfide (D) in case of (1).

\begin{tabular}{|c|c|c|c|c|}
\hline mixing rates & A & B & C & D \\
\hline $1: 1(\mathrm{C} \& D)$ & 5 & 2 & 7 & 4 \\
\hline $1: 5(\mathrm{C} \& D)$ & 4 & 0 & 5 & 9 \\
\hline $5: 1(\mathrm{C} \& D$ & 3 & 0 & 6 & 9 \\
\hline
\end{tabular}




\section{EXPERIMENTAL DISCUSSION}

In order to improve the experimental results given in Table V, we use a genetic algorithm (GA) to find a suitable value $\alpha$. Component of mixed odors has changed as shown in Fig. 15 where red bars are the previous classification result. Blue bars are the classification result of after improvement. However, we could not be achieved the eventual goal that we correctly classify the components of mixed odors. We have to consider more details of GA to improve the accuracy of the classification. Future, we review the evaluation part and the choice of the parent. In addition, we assumed that classification accuracy improves by performing the removal per sensor.

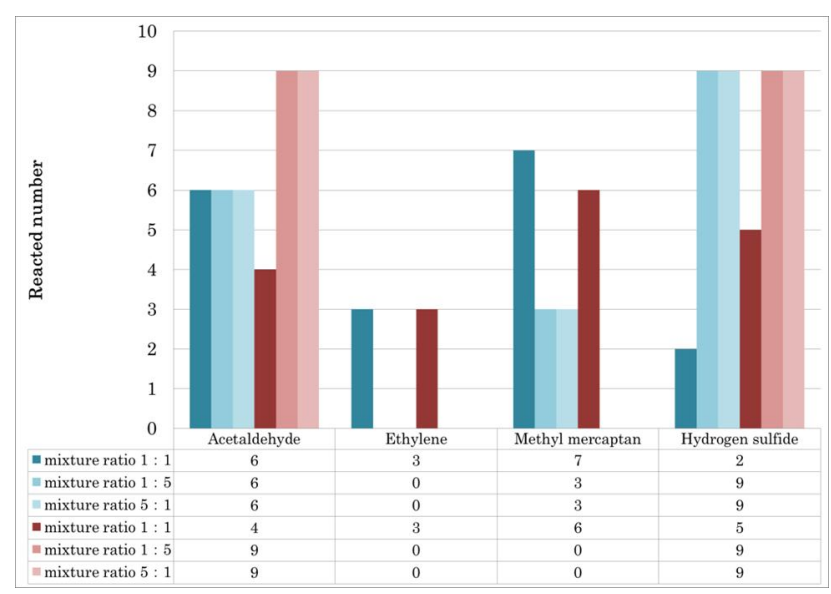

Fig. 15 - Results using GA to find $\alpha$

\section{CONCLUSIONS}

We considered a method to classify the mixed odors into original odor before mixing using a neural network. In that case the classification boundary becomes bigger world. Therefore, we have proposed one way to classify the mixed odors classification from single classification neural network. By modifying the features by using (1) it may be possible. But we must find the suitable coefficient $\alpha$. We have considered to use a genetic algorithm to determine the suitable coefficient. Since there are many approaches to find the $\alpha$, we will compare with the different approaches.

\section{ACKNOWLEDGEMENTS}

This research has been supported by Grant-inAid for Scientific Research for (B) No. 24360141and Technology(JST), and Japan Keirin and Autorace (JKA) Foundations2017M144.

\section{REFERENCES}

[1] L. Buck and R. Axel, "A novel multigene family may encode odorant receptors: a molecular basis for odor recognition", Cell, 65, 175-187, 1991.

[2] L. Buck, "Information coding in the vertebtrate olfactory system", Annu. Rev. Neurosci., 19, 517-544, 1996.

[3] J. Todrank, C. J. Wysocki, and G. K. Beauchamp, "The effects of adaptation on the perception of similar and dissimilar odors", Chem. Senses, 16, 467-482, 1991.

[4] W. S. Cain and E. H. Polak, Olfactory adaptation as an aspect of odor similarity, Chem. Senses, 17, 481-491, 1992.

[5] W. S. Cain and B. C. Potts, Switch and bait: probing the discriminative basis of odor identification via recognition memory, Chem. Senses, 21, 35-44, 1996.

[6] K.J. Rossiter, "Structure-odor relationships", Chem. Rev. , 96, 3201-3240, 1996.

[7] P. Callegari, J. Rouault, and P. Laffort, "Olfactory quality from descriptor profiles to similarities", Chem. Senses, 22, 1-8, 1997.

[8] M. L. Laska and D. Freyer, "Olfactory discrimination ability for aliphatic esters in squirrel monkeys and humans", Chem. Senses, 22, 457-465, 1997.

[9] Laska M. and P. Tuebner, "Olfactory discrimination ability of human subjects for ten pairs of enantiomers", Chem. Senses, 24, 161170, 1999.

[10] P. M. Wise and W. S. Cain, "Latency and accuracy of discriminations of odor quality between binary mixtures and their components", Chem. Senses, 25, 247-265, 2000.

[11] D. A. Stevens and R. J. O'Connell, "Semanticfree scaling of odor quality", Physiol. Behav. , 60, 211-215, 1996.

[12] F. Wang, A. Nemes, M. Mendelsohn, and R. Axel, "Odorant receptors govern the formation of a precise topographic map", Cell, 93, 47-60, 1998.

[13] M. Yokoi, K. Mori, and N. Shigetada, "Refinement of odor molecule tuning by dendrodendritic synaptic inhibition in the olfactory bulb", Proc. Natl Acad. Sci. USA, 92, 3371-3375, 1995.

[14] N. Baric, M. Bucking, and M. Rapp, "A novel electronic nose based on minimized saw sensor arrays coupled with some enhanced headspace analysis and its use for rapid determination of volatile organic compounds in food quality monitoring," Sensors and Actuator B, vol. 114, 482-488, 2006.

[15] A. Norman, F. Stam, A. Morrissey, M. Hirschfelder, and D. Enderlein, "Packaging effects of a novel explosion-proof gas sensor", Sensors and Actuator B, vol. 114, 287-290, 2003. 
[16] J. Ruther, T.Meiners, and J. M. Steidlem, "Rich in phenomena-lacking in terms. A classification of kairomones", Chemoecology, vol. 12, 161$167,2002$.

[17] M. Ghasemi-Varnamkhasti, S. Saeid and M. M. Siadat,, "Biomimetic-based odor and taste sensing systems to food quality and safety characterization: An overview on basic principles and recent achievements", Journal of Food Engineering, , vol. 100, 377-387, 2010.

[18] S. Marco; Agustín Gutierrez-Galvez, "Signal and data processing for machine olfaction and chemical sensing: A review", IEEE Sensors Journal, vol. 12, 3189 - 3214.

[19] R. Young, W. Buttner, B. Linnel, and R. Ramesham, "Electronic nose for space program applications", Sensors and Actuator B, vol. 93, 7-16, 2003.

[20] J. A. Milke, Application of neural networks for discriminating fire detectors, 1995, international Conference on Automatic Fire Detection, AUBE'95, 10th, Duisburg, Germany.

[21] T. Fujinaka, M. Yoshioka, S. Omatu, and T. Kosaka, "Intelligent electronic nose systems for fire detection systems based on neural networks", International Journal of Advances in Intelligent Systems, vol. 2, 268-277, 2009.

[22] S. Omatu and M. Yano, "Mixed odors classification by neural networks", The 8th
IEEE International Conference on Intelligent Data Acquisition and Advanced Computing Systems: Technology and Applications, 24-26 September 2015, Warsaw, Poland, 24-26.

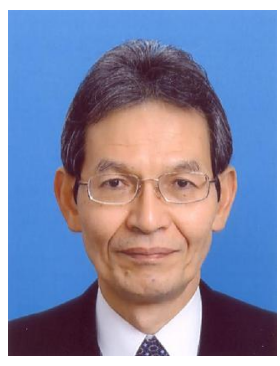

Sigeru Omatu. He is Professor of at the Department of System Design Engineering, Faculty of Robotics \& Design Engineering, Osaka Institute of Technology, Osaka, Japan. He received his Ph.D. in Electronic Engineering from Osaka Prefecture University and joined the faculty at University of Tokushima in 1969. He was Professor of University of Tokushima in 1988 and Professor of Osaka Prefecture University in 1995. He has been Professor of Osaka Institute of Technology since 2010. His honors and awards include the Best Paper Awards for Distributed Parameter System Theory, IEE of Japan, 1991, for Intelligent Classification, JSME, 1995, for Coin and Bill Classification, SICE, Japan, 1995, for Intelligent Smell Classification,IARIA, 2008, for Neuro-Control, IARIA, 2009. He received Ichimura Distinguished Award in 1996 and Science Award for Research Sector from Commendation for Science and Technology by the Minister of Education, Culture, Sports, Science and Technology in 2011. 